\title{
Stellar wind measurements for Colliding Wind Binaries using X-ray observations
}

\author{
Yasuharu Sugawara ${ }^{1}$, Yoshitomo Maeda ${ }^{1}$ and Yohko Tsuboi ${ }^{2}$ \\ ${ }^{1}$ Institute of Space and Astronautical Science, Japan Aerospace Exploration Agency, \\ 3-1-1 Yoshinodai, Chuo-ku, Sagamihara, Kanagawa 229-8510, Japan \\ email: sugawara.yasuharu@jaxa.jp \\ ${ }^{2}$ Department of Physics, Faculty of Science \& Engineering, Chuo University, \\ 1-13-27 Kasuga, Bunkyo, Tokyo 112-8551, Japan
}

\begin{abstract}
We report the results of the stellar wind measurement for two colliding wind binaries. The X-ray spectrum is the best measurement tool for the hot postshock gas. By monitoring the changing of the the X-ray luminosity and column density along with the orbital phases, we derive the mass-loss rates of these stars.
\end{abstract}

Keywords. binaries: spectroscopic, stars: Wolf-Rayet, X-rays: stars, stars: mass loss

\section{Introduction}

Mass-loss is one of the most important and uncertain parameters in the evolution of a massive star. Colliding wind binary is the best testing ground for plasma shock physics, because plasma properties vary with binary separations. We have reported an approach to determine a mass-loss rate of a binary system with a highly eccentric orbit with Xray multi-phase spectroscopy (Sugawara et al. 2015). In this paper. we select WR21a $\left(\mathrm{O} 3 / \mathrm{WN} 5 \mathrm{ha}+\mathrm{O} 3 \mathrm{Vz}\left(\left(\mathrm{f}^{*}\right)\right)\right)$ and WR25 (WN6h+O4f) as samples.

\section{Wind measurements}

In the case of WR21a, we fitted the X-ray spectra from XMM-Newton with two absorbed thin thermal plasma model. As periastron approached, the local column density increased, which can be explained as self-absorption by the W-R wind. Therefore, the local column density (mass-loss rate) can be expressed as the integral from the X-ray emitting region (a compact region around periastron) to infinite distance along the line of sight. Using the column densities from spectral fitting, our estimated mass-loss rate for primary star of WR21a is $(5.5 \pm 1.0) \times 10^{-6} \mathrm{M}_{\odot} \mathrm{yr}^{-1}$. This is consistent the results using the similar method (Gosset \& Nazé 2016).

In the case of WR25, we used the reported local column density data measured by XMM-Newton (Pandy et al. 2014). The column density data could be reproduced by the model using low eccentricity value $(e=0.35)$ and low inclination angle $\left(i<10^{\circ}\right)$. Our estimate for W-R (WN) star is $5.1 \times 10^{-5} \mathrm{M}_{\odot} \mathrm{yr}^{-1}$. By using this method for WR25, we could limit not only mass-loss rate but also orbital inclination angle.

This work was supported by JSPS KAKENHI Grant Number JP16K17667.

\section{References}

Gosset, E. \& Nazé, Y. 2016, A\& A, 590, A113

Pandey, J. C., Pandey, S. B., \& Karmakar, S. 2014, ApJ, 788, 84

Sugawara, Y., Maeda, Y., Tsuboi, Y., et al. 2015, PASJ, 67, 121 